\title{
Wie können heterogene Prozessdaten automatisch in einem Energiemanage- mentsystem zusammengeführt werden?
}

\author{
Maxim Runge ${ }^{1}$, Leif-Thore Reiche ${ }^{2}$, Prof. Dr. Karl-Heinz Niemann ${ }^{3}$, Prof. Dr. \\ Alexander Fay ${ }^{4}$
}

\section{Zusammenfassung}

Mit der Anwendung der Norm ISO 50001 und der einhergehenden Einführung eines Energiemanagementsystems (kurz EnMS) kann eine sukzessive Erhöhung der Energieeffizienz erreicht werden. Zur Umsetzung von Energie-Monitoring- oder Standby-Management-Funktionalitäten müssen Energiedaten in der Feldebene bereitgestellt werden und auf Edge-Devices oder SPSen mittels eines Energiemanagement-Programms ggf. im Datenformat angepasst, skaliert und auf eine etablierte Kommunikationsschnittstelle (z.B. basierend auf OPC UA- oder MQTT) abgebildet werden. Die Erstellung dieser Energiemanagement-Programme geht mit einem hohen Engineering-Aufwand einher, denn die Feldgeräte aus der heterogenen Feldebene stellen die Energiedaten nicht in einer standardisierten Semantik bereit. Um diesem Engineering-Aufwand entgegenzuwirken, wird ein Konzept für ein universelles Energiedateninformationsmodell (kurz UEDIM) vorgestellt. Dieses Konzept sieht die Bereitstellung der Energiedaten an das EnMS in einer semantisch standardisierten Form vor. Zur weiteren Entwicklung des UEDIM wird im Beitrag näher untersucht, in welcher Form Energiedaten in der Feldebene bereitgestellt werden können und welche Anforderungen für das UEDIM aufzustellen sind.

\section{Stichwörter}

Energiemanagement, Informationsmodell, Anforderungsermittlung

\section{Einleitung}

Die Einführung eines Energiemanagementsystems (kurz EnMS) auf Basis der Norm ISO 50001 [1] in einem industriellen Produktionsbetrieb kann eine kontinuierliche Erhöhung der Energieeffizienz bewirken. Nach [2] werden die für den Betrieb eines EnMS erforderlichen Soft- und Hardwarekomponenten unter dem Begriff technisches Energiemanagement (kurz tEnMS) zusammengefasst. Die Komponenten eines tEnMS dienen zum Sammeln, Kommunizieren, Speichern, Bewerten und Anzeigen von Messdaten, die z.B. für Monitoring-Anwendung verwendet werden können. Des Weiteren zählen zum tEnMS auch Geräte, die mit Hilfe sogenannter Lastmanagementfunktionen in energiesparende Betriebszustände versetzt werden können. Zur Realisierung von Energie-Monitoring- und Lastma-

\footnotetext{
${ }^{1}$ M. Eng. Maxim Runge, Hochschule Hannover, Hannover

${ }^{2}$ M. Sc. Leif-Thore , Helmut-Schmidt-Universität / Universität der Bundeswehr, Hamburg

${ }^{3}$ Prof. Dr.-Ing. Karl-Heinz Niemann, Hochschule Hannover, Hannover

${ }^{4}$ Prof. Dr.-Ing. Alexander Fay, Helmut-Schmidt-Universität / Universität der Bundeswehr, Hamburg 
nagement-Funktionalitäten ist ein Datenaustausch zwischen den Feldgeräten und dem Energiemanagementsystem erforderlich. Diese Daten werden im Folgenden als Energiemanagement-Informationen (kurz EmI bezeichnet.

Im Rahmen des tEnMS können EmI durch verschiedene Feldgerätetypen bereitgestellt werden. Diese Feldgerätetypen sind einerseits, z.B. als OPC UA-fähiges Gerät aus dem Industrial Internet of Things (IIoT-Gerät), zur direkten Kommunikation mit einer Energiemanagement-Applikation geeignet. Andererseits stellen etablierte Gerätetypen die EmI über Feldbussysteme, Punkt-zu-Punkt-Verbindungen wie IO-Link oder andere Kommunikationssysteme bereit. Diese EmI werden aus der Feldebene an ein Edge-Device oder eine SPS übertragen und müssen mittels eines Energiemanagement-Programms ggf. im Datenformat angepasst, skaliert und auf eine geeignete Kommunikationsschnittstelle (z.B. eine OPC UA-Schnittstelle) abgebildet werden. Die Erstellung dieser Energiemanagement-Programme geht nach [3] mit einem hohen Engineering-Aufwand einher, denn die Feldgeräte aus der heterogenen Feldebene stellen die EmI nicht in einer standardisierten Form bereit. Bei der Erstellung des Energiemanagement-Programms muss die Semantik, in der die EmI in Rohdaten auf dem Edge-Device oder der SPS eintreffen, den individuellen Gerätehandbüchern entnommen werden. Auf Grundlage dieser Semantik muss ermittelt werden, welche Verarbeitungsschritte für die jeweiligen EmI anzuwenden sind, damit diese in die Energiemanagement-Applikation eingebunden werden können.

Um diesem Aufwand entgegenzuwirken, wird in den Beiträgen [4] und [5] ein Konzept für ein universelles Energiedateninformationsmodell (kurz UEDIM) vorgestellt. Dieses Konzept sieht die Feldgerätetyp-unabhängige Bereitstellung der EmI an die Energiemanagement-Applikation in einer semantisch standardisierten Form vor.

In Kapitel 2.1 wird für die Anforderungsermittlung eines UEDIM relevante Literatur vorgestellt. Nachfolgend wird in Kapitel 3 das Konzept eines UEDIM und dessen Platzierung in der Kommunikationshierarchie beschrieben. Ergänzend wird das Vorgehen bei der Ableitung von Anforderungen auf Grundlage der durchgeführten Recherche dargestellt. Der Beitrag wird mit einer Zusammenfassung der präsentierten Inhalte und einem Ausblick der nächsten Arbeitsschritte im zugehörigen Forschungsprojekt „Entwicklung von Energiemanagementschnittstellen für IoT-Technologien“ abgeschlossen.

\section{Stand der Technik}

Um Anforderungen für ein UEDIM aufstellen zu können, wird in diesem Kapitel erfasst, welche Feldgeräte zur Bereitstellung von EmI geeignet sind. Nachfolgend wird näher untersucht, wie EmI heutzutage an eine Energiemanagement-Applikation übertragen werden können. Abschließend wird ein Ausblick gegeben, welche Möglichkeiten für die Bereitstellung von EmI es in Zukunft gibt.

\subsection{Bestandsaufnahme geeigneter Geräte für die Übernahme von Energiemanagement-Aufgaben}

Nach [5] können in der Feldebene verschiedene Ethernet-basierte und nicht-Ethernet-basierte Gerätetypen zur Übernahme von Energiemanagement-Aufgaben eingesetzt werden. Bild 1 zeigt beispielhaft, dass die Feldebene heterogen charakterisiert sein kann und verschiedene Feldgerätetypen mit unterschiedlichen Kommunikationsschnittstellen zur Bereitstellung von EmI eingesetzt werden können. Nicht-Ethernet-basierte Gerätetypen können z.B. IO-Link-Geräte wie Stromüberwachungsrelais oder PROFIBUS-basierte Frequenzumrichter sein, die für das Monitoring relevante EmI (beispielsweise Strom, Leistungsfaktor oder Energiezählwerte) bereitstellen können. Ethernet-basierte Gerätetypen verfügen über Kommunikationsschnittstellen wie beispielsweise Modbus TCP oder PROFINET. Einige Ethernet-basierte Gerätetypen unterstützen so genannte Energieprofile. Diese sind jeweils auf 
ein spezifisches Kommunikationsprotokoll ausgerichtet. Solche Energieprofile sind PROFIenergy [6] für PROFINET, sercos Energy [7] für sercos III oder CIP Energy [8] für DeviceNet und EtherNet/IP. Die Energieprofile unterstützen tEnMS-Funktionalitäten aus den Bereichen Messdatenerfassung (z. B. Energiezählwerte) und Lastmanagement (z.B. Standby-Management). Mit Hilfe der Energieprofile können ausgehend vom Feldgerät die EmI im Rahmen der jeweiligen Energieprofilspezifikation in standardisierter Form einem Edge-Device oder einer SPS bereitgestellt werden, sodass die tEnMSFunktionalitäten für überlagerte Systeme verfügbar gemacht werden. Die EmI der genannten Ethernet-basierten und nicht-Ethernet-basierten Gerätetypen liegen in unterschiedlicher Semantik auf dem Edge-Device oder der SPS vor und stehen zur Weiterverarbeitung durch ein Energiemanagement-Programm und für die Bereitstellung der EmI zur Energiemanagement-Applikation zur Verfügung. Ethernet-basierte IIoT-Geräte weisen i. d. R. eine geeignete Kommunikationsschnittstelle (z.B. OPC UA) auf, die eine direkte Kommunikation mit der Energiemanagement-Applikation ermöglicht. Die Aufbereitung und Bereitstellung der EmI gegenüber der Energiemanagement-Applikation wird im nächsten Abschnitt näher erläutert.

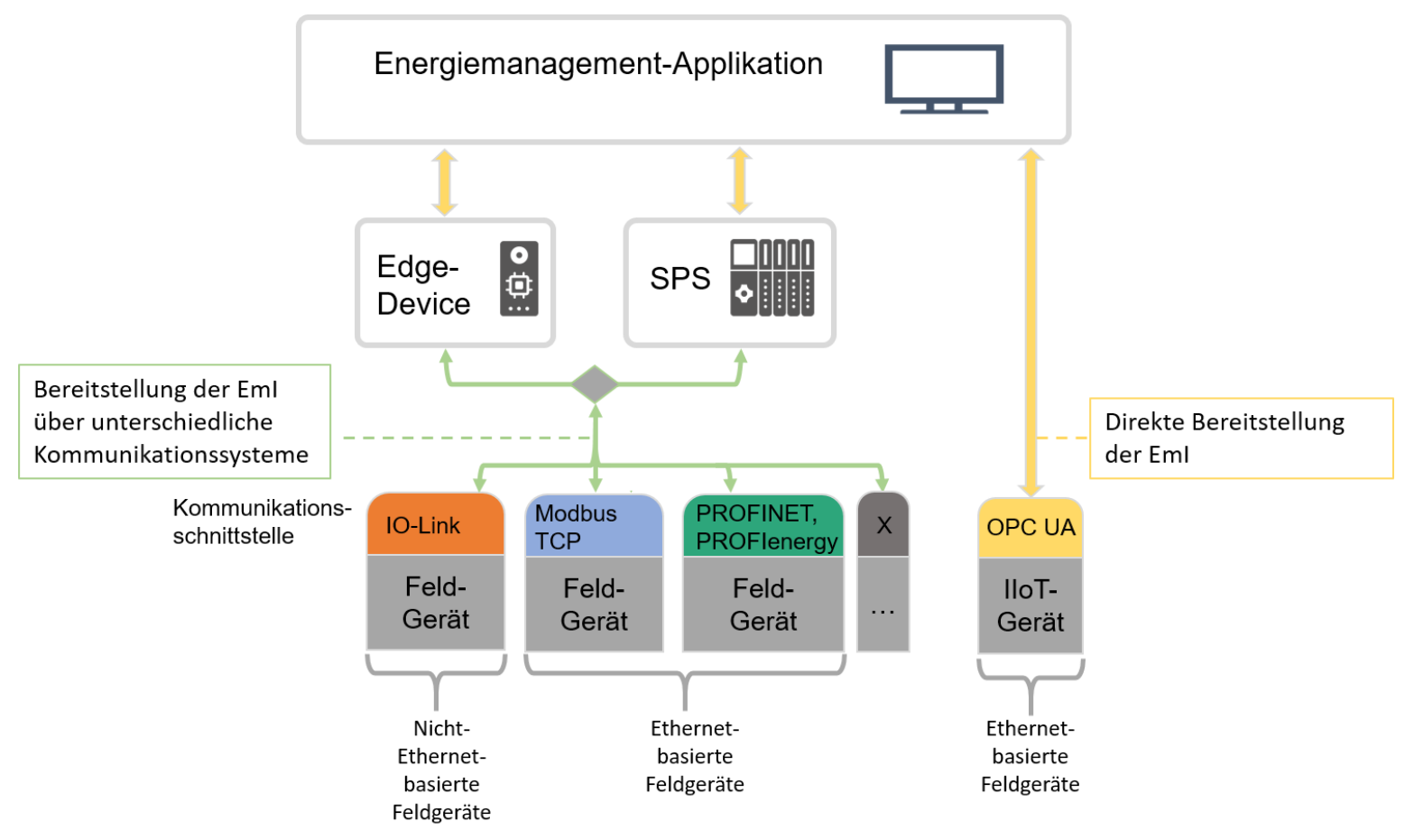

Bild 1: Überblick über die heterogene Feldebene

\subsection{Austausch der EmI mit der Energiemanagement-Applikation}

Bild 2 zeigt, dass die EmI der unterschiedlichen Gerätetypen aus der Feldebene im Rohdatenformat auf den Geräten 1, 2 und 3 vorliegen, die zur Kommunikation mit der Energiemanagement-Applikation geeignet sind. Die EmI müssen mittels eines Energiemanagement-Programms ggf. im Datenformat angepasst und skaliert werden. Im Rahmen eines erforderlichen Daten-Mappings können die EmI auf Variablenstrukturen übertragen werden, die auf eine geeignete Kommunikationsschnittstelle, wie eine OPC UA- oder MQTT-Schnittstelle, abgebildet werden. Bei der Programmerstellung können unterschiedliche Konzepte bezüglich der semantischen Aufbereitung der EmI verfolgt werden. In Konzept (A) werden manuell OPC UA Variablen angelegt, die zur Bereitstellung der EmI genutzt werden. Die 
Variablenbezeichnungen sowie zugeordneten Datentypen können beispielsweise einem Firmen-internen Standard oder den individuellen Vorgaben der programmerstellenden Person folgen. Angelehnt an Konzept (A) erfolgt bei Konzept (B) ebenso eine individuelle Deklaration von Variablen, die für die Bereitstellung der EmI über eine MQTT-Schnittstelle durchgeführt werden muss. In den Konzepten (A)

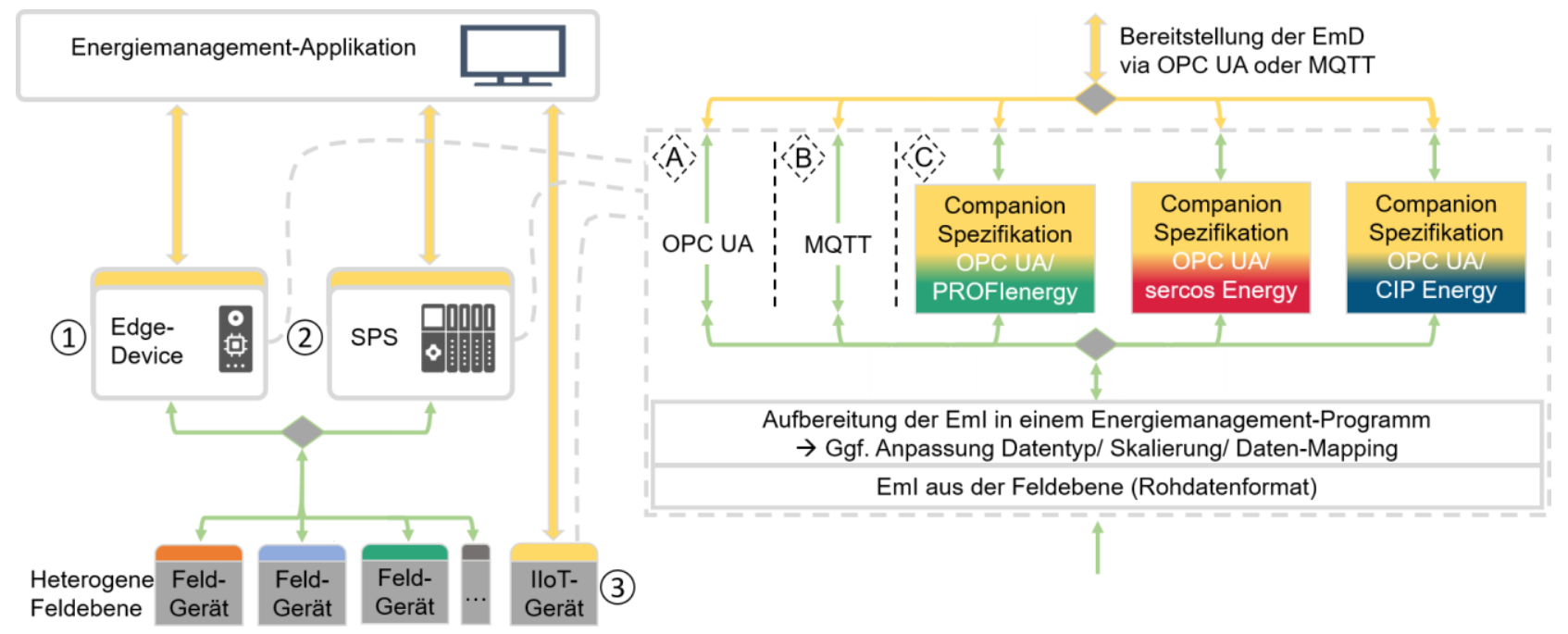

Bild 2: Bereitstellung der EmI an die Energiemanagement-Applikation

und (B) werden die EmI individuell von der programmerstellenden Person aufbereitet, sodass eine nicht standardisierte Semantik vorliegt. Konzept (C) sieht die Nutzung einer OPC UA Companion Spezifikation vor. Mittels Import eines sogenannten OPC UA Node-Schemas wie [9] werden zum Anlegen von OPC UA-Variablen Klassenstrukturen bereitgestellt. Mit der Instanziierung dieser Klassenstrukturen werden automatisch OPC-UA-Variablen angelegt, die z.B. Bezeichnungen und Datentypen der Variablen für die Darstellung eines Energiemesspunkts festlegen. Das Konzept (C) umfasst die Gewährleistung einer standardisierten Semantik mit der Anwendung einer OPC UA Companion Spezifikation. In OPC UA Companion Spezifikationen werden entsprechende Informationsmodelle, die z.B. auf Basis eines Energieprofils erstellt wurden, mit standardisierten OPC UA-Elementen im Format des OPC UAAdressraummodells [10] dargestellt. Die im Rahmen der Bereitstellung der EmI relevanten OPC UA Companion Spezifikationen sind dabei auf Energieprofile wie PROFIenergy, sercos Energy oder CIP Energy ausgerichtet. Für PROFIenergy wurde bereits eine OPC Companion Spezifikation [11] veröffentlicht. Im Fall des Energieprofils sercos Energy wurde eine allgemeine OPC UA Companion Spezifikation [12] veröffentlicht, die auf das Bussystem sercos III ausgerichtet ist. Für das Kommunikationssystem CIP ist die Entwicklung einer allgemeinen OPC UA-Companion Spezifikation angekündigt [13]. für die genannten Energieprofile entwickelt. Die Semantik der EmI orientiert sich dabei an dem jeweils zugeordneten Energieprofil, sodass kein allgemeingültiger Standard besteht.

\subsection{Möglichkeiten der Bereitstellung von EmI in der Zukunft}

Neben den bisherigen Möglichkeiten, EmI bereitzustellen, werden aktuell und zukünftig neue Lösungen ausgearbeitet und bestehende Lösungen erweitert, da die bisherige Übertragung von EmI erhöhte Engineering-Aufwände in der Erstellung von Energiemanagement-Programmen mit sich zieht. [2] Nachfolgend werden Möglichkeiten beschrieben, wie die zukünftige Bereitstellung von EmI erfolgen kann.

Nach [14] kann die Einführung oder der Ausbau eines tEnMS nach einem parallelen oder nach einem integrierten Ansatz erfolgen. Beim parallelen Ansatz werden z.B. neue Messgeräte in den vorhande- 
nen Automatisierungsaufbau eingebracht, die EmI wie Energiezählwerte für Monitoring-Anwendungen liefern können. Der integrierte Ansatz sieht vor, dass bereits zur Anlagenautomatisierung erforderliche Geräte wie z.B. Frequenzumrichter genutzt werden, um EmI wie Energiezählwerte für Monitoring-Anwendungen liefern zu können. Somit fallen bei diesem Ansatz keine Anschaffungskosten für Neugeräte an. Die Umsetzung des integrierten Ansatzes erfordert die Erstellung von neuen Programmteilen auf Edge-Devices oder der SPSen. Diese Programmteile sind erforderlich, um EmI wie z.B. Energiezählwerte aus Feldgeräten abzurufen oder um Lastmanagement-Befehle an Feldgeräte weiterleiten zu können. Liegen die EmI in einem Energieprofil-basierten Datenformat auf der Steuerungsebene vor, können aufgrund der standardisierten Semantik Codegenerator-Anwendungen genutzt werden, um dem Engineering-Aufwand bei der Erstellung von entsprechenden Energiemanagement-Programmen entgegenzuwirken. Das Konzept des Beitrags [14] sieht außerdem vor, dass eine automatische Generierung des OPC UA-Adressraums realisiert wird und EmI aufwandsarm der Energiemanagement-Applikation bereitgestellt werden können.

In Beitrag [15] wird angeführt, dass in der heutigen heterogenen Feldebene viele verschiedene Feldbusse und Kommunikationssysteme eingesetzt werden und diese jeweils auf einem eigenem Informationsmodell basieren. Im genannten Beitrag wird diesbezüglich das Konzept vorgestellt, auch Feldgeräte in das OPC UA-Kommunikationssystem zu integrieren. Der Vorteil der standardisierten Semantik der OPC UA-Kommunikation ist im Hinblick auf eine skalierbare Interoperabilität für Industrie 4.0Anwendungen maßgeblich. [15]

Neben OPC UA ist MQTT ein alternatives IIoT-Protokoll, das zur Bereitstellung von EmI eingesetzt werden kann [16]. Der Einsatz eines MQTT-Kommunikationssystems ist im direkten Vergleich mit OPC UA nicht auf eine standardisierte Semantik ausgerichtet. Der Schwerpunkt bei MQTT liegt auf der effizienten Übertragung von Daten (,,lightweight protocol“). Die Vorteile des Protokolls liegen in der sicheren Datenübertragung bei geringer Netzwerkqualität mit Verbindungsabbrüchen, geringer Netzwerkbandbreite oder einer hohen Latenz. [17]

In der NE 176 [18] beschreibt der Verein Interessengemeinschaft Automatisierungstechnik der Prozessindustrie e.V. (kurz NAMUR) ein Informationsmodell, das bei Anwendungen aus dem Bereich „Monitoring und Optimierung“ eingesetzt werden kann. Diese NAMUR-Empfehlung umfasst die Definition eines standardisierten Informationsmodells, welches eine einheitliche Semantik für Produktions- und Anlagendaten bereitstellt. Die einheitliche Semantik wird über eine standardisierte OPC UASchnittstelle realisiert. Den bereitzustellenden EmI werden jeweils eindeutige semantische Kennungszahlen (kurz „ID") zugeordnet. Für die Kennungszahlen sind in der Standardisierung Attribute wie „Name, Datentyp, Maßeinheit, zulässiger Wertebereich und weitere Attribute (Metadaten)“ definiert. [18]

\section{Lösungsansatz zur Vereinheitlichung der heterogen vorliegenden EmI}

In diesem Kapitel wird ein Konzept für ein UEDIM vorgestellt, welches eine semantische Standardisierung der EmI gewährleisten soll. Nachfolgend werden auf Basis von Kapitel 2.1 Anforderungen an ein UEDIM abgeleitet.

\subsection{Konzept für ein UEDIM}

Die Untersuchung der Gerätetypen hinsichtlich der Semantik der EmI in Kapitel 2.1 hat ergeben, dass die Feldebene heterogen aufgebaut ist. Um eine Bereitstellung der EmI gegenüber Energiemanagement-Applikationen in standardisierter Semantik zu erreichen, wird ein UEDIM konzipiert, das die EmI der heterogenen Feldebene strukturieren kann. 
Bild 3 zeigt die Platzierung des UEDIM in der Kommunikationshierarchie. In den Fällen (A-D) werden die EmI von Ethernet-basierten und nicht-Ethernet-basierten (1) Feldgeräten über das jeweilige Kommunikationssystem (2) den spezifischen Kommunikationsschnittstellen (3) des Edge-Devices oder der SPS bereitgestellt. Auf der Steuerungsebene können die EmI auf das UEDIM abgebildet werden, indem die SPS oder das Edge-Device über ein entsprechendes Programm eine Umrechnung der proprietär eintreffenden Daten auf das UEDIM vornimmt (4). EmI, die auf dem Datenformat eines standardisierten Energieprofils beruhen, können mit Hilfe der spezifisch auf das jeweilige Energieprofil ausgerichteten OPC UA Companion Spezifikation im UEDIM abgebildet werden.

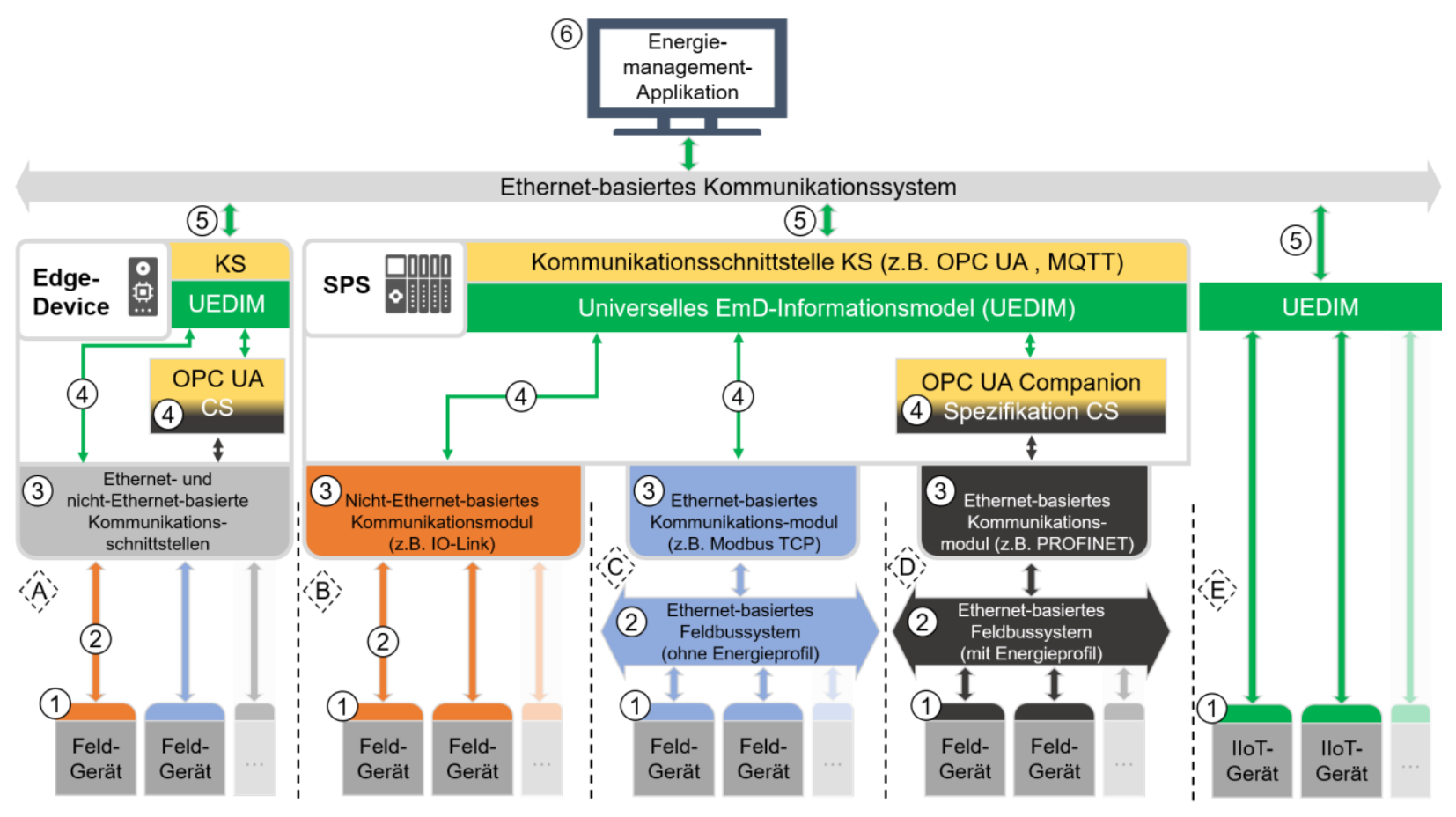

Bild 3: Platzierung des UEDIM in der Kommunikationshierarchie, basierend auf [5]

In der standardisierten Semantik des UEDIM können die EmI über geeignete Kommunikationsschnittstellen (5), die auf OPC UA oder MQTT basieren können, der Energiemanagement-Applikation (6) bereitgestellt werden. In Fall (E) können IIoT-Devices eine direkte Bereitstellung der EmI in der standardisierten Semantik des UEDIM, z.B. über OPC UA- oder MQTT-Kommunikationsschnittstellen realisieren.

\subsection{Anforderungen an ein UEDIM}

Bei der Ableitung von Anforderungen an ein UEDIM auf Basis des Standes der Technik werden sogenannte Persona-Profile [19] entworfen, um die Bedürfnisse der Stakeholder tabellarisch zu erfassen. Die entworfenen Personas haben aufgrund der spezifischen Aufgaben, z.B. bei der Edge-Device-Programmentwicklung, direkte Schnittpunkte mit dem UEDIM oder den standardisiert bereitgestellten EmI.

In Tabelle 1 wird eines der entworfenen Persona-Profile beispielhaft abgebildet.

Tabelle 1: Persona-Profil Programm-Entwickler*in für Edge-Devices und SPSen

\section{\begin{tabular}{|l|ll}
\hline Arbeitsrolle & $-\quad$ Programm-Entwickler*in für Edge-Devices und SPSen
\end{tabular}}




\begin{tabular}{|c|c|}
\hline Kenntnisse & $\begin{array}{ll}- & \text { Erfahrung im Umgang mit den eingesetzten Entwicklungsumgebungen } \\
- & \text { Kenntnisse bei der Implementierung von Kommunikationsbausteinen zur } \\
& \text { Geräteanbindung (z.B. mittels Anwendung von Energieprofilen) } \\
- & \text { Edge-Device-/ SPS-Programmentwicklung } \\
- & \text { Hardwarekonfiguration } \\
- & \text { EmI-Mapping auf dem Edge-Device/ auf der SPS }\end{array}$ \\
\hline Ziele & 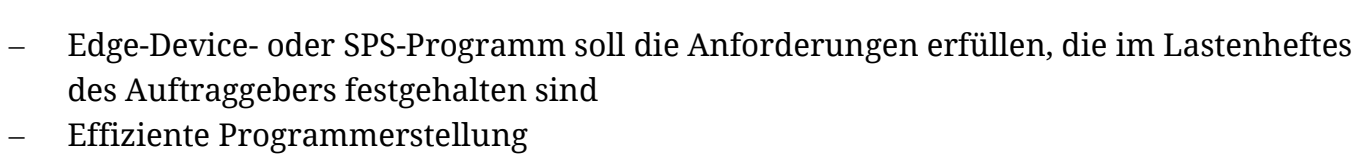 \\
\hline Arbeitsprozesse & $\begin{array}{ll}- & \text { Erstellung von Edge-Device- oder SPS-Programmen } \\
- & \text { Änderung von Edge-Device- oder SPS-Programmen } \\
- & \text { Inbetriebnahme } \\
- & \text { Fehlersuche und Diagnose }\end{array}$ \\
\hline Wünsche & $\begin{array}{l}\text { - } \quad \text { Reduzierung des zu erbringenden Engineering-Aufwandes } \\
\text { - } \quad \text { Zugriff auf möglichst wenige Geräte-Dokumentationen und -Handbücher zur Planung/ } \\
\text { Umsetzung }\end{array}$ \\
\hline
\end{tabular}

In Form von Persona-Profilen werden weitere Personengruppen erfasst, die von dem Einsatz des UEDIM profitieren können. Tabelle 2 gibt einen Überblick über die entworfenen Personas und die Einteilung in Persona-Gruppen. Hinzukommend wird den Personas eine Priorität zugeordnet, die eine Einschätzung der Autoren darüber angibt, welche Personas im Rahmen ihrer Tätigkeit direkte Berührungspunkte mit einem UEDIM aufweisen.

Tabelle 2: Gruppierung der Personas

\begin{tabular}{|c|c|c|c|}
\hline Persona-Gruppe & $\begin{array}{l}\text { Persona } \\
\text { Nr. }\end{array}$ & Persona-Bezeichnung & Priorität \\
\hline Geräteentwicklung & $\begin{array}{l}1 \\
2\end{array}$ & $\begin{array}{ll}- & \text { Gerätehersteller*in für Feldgeräte } \\
- & \text { Gerätehersteller*in für Edge-Devices und SPSen }\end{array}$ & $\begin{array}{ll}- & \text { Hoch } \\
- & \text { Hoch }\end{array}$ \\
\hline $\begin{array}{l}\text { Anlagenerrichtung und } \\
\text { Inbetriebnahme }\end{array}$ & $\begin{array}{l}3 \\
4 \\
5\end{array}$ & $\begin{array}{ll}- & \text { Entwicklungsingenieur*in für Produktionsanlagen } \\
- & \text { Programm-Entwickler*in für Edge-Devices und } \\
& \text { SPSen } \\
- & \text { Systemkonfigurator*in für Prozessleitebene und } \\
& \text { MES }\end{array}$ & $\begin{array}{ll}- & \text { Hoch } \\
- & \text { Hoch } \\
- & \text { Hoch }\end{array}$ \\
\hline Prozessleitebene & 6 & - $\quad$ Personal in der Leitwarte & - $\quad$ Mittel \\
\hline Betrieb & $\begin{array}{l}7 \\
8 \\
9\end{array}$ & $\begin{array}{ll}- & \text { Betriebsleiter*in } \\
- & \text { Anlagenbediener*in } \\
- & \text { Energiemanager*in }\end{array}$ & $\begin{array}{ll}- & \text { Mittel } \\
- & \text { Gering } \\
- & \text { Hoch }\end{array}$ \\
\hline Einkauf & $\begin{array}{l}10 \\
11\end{array}$ & $\begin{array}{ll}- & \text { Einkäufer*in für Energie } \\
- & \text { Einkäufer*in (Maschinen und Einzelkomponenten) }\end{array}$ & 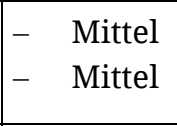 \\
\hline $\begin{array}{l}\text { Instandhaltung und } \\
\text { Optimierung } \\
\end{array}$ & 12 & - Instandhalter*in & - $\quad$ Mittel \\
\hline Management & 13 & - Geschäftsführung & - $\quad$ Mittel \\
\hline
\end{tabular}




\begin{tabular}{|c|c|c|c|}
\hline Persona-Gruppe & $\begin{array}{l}\text { Persona } \\
\text { Nr. }\end{array}$ & Persona-Bezeichnung & Priorität \\
\hline $\begin{array}{l}\text { Dienstleister } \\
\text { Energiemanagement- } \\
\text { Sektor (betriebsextern) }\end{array}$ & $\begin{array}{l}14 \\
15 \\
16\end{array}$ & $\begin{array}{ll}- & \text { Programm-Entwickler*in für Edge-Devices und } \\
& \text { SPSen (extern) } \\
- & \text { Systemkonfigurator*in für Prozessleitebene und } \\
& \text { MES (extern) } \\
- & \text { Instandhalter*in (extern) }\end{array}$ & $\begin{array}{ll}- & \text { Hoch } \\
- & \text { Hoch } \\
- & \text { Mittel }\end{array}$ \\
\hline
\end{tabular}

Auf der Grundlage der entworfenen Personas werden, basierend auf [5] und anhand der Inhalte von Kapitel 2.1, Anforderungen an ein UEDIM abgeleitet und in Tabelle 3 aufgeführt. Die Anforderungen werden den entworfenen Personas zugeordnet.

Tabelle 3: Anforderungen an ein UEDIM, basierend auf [5]

\begin{tabular}{|c|c|c|}
\hline Anforderungsgruppe & $\begin{array}{l}\text { Zugeordnete } \\
\text { Personas }\end{array}$ & Funktionale (f) und nicht-funktionale (nf) Anforderungen \\
\hline \multirow[t]{4}{*}{$\begin{array}{l}\text { Interne Strukturierung der } \\
\text { EmI im UEDIM }\end{array}$} & $\begin{array}{l}1,2,3,4,5,14, \\
15\end{array}$ & $\begin{array}{l}\text { Standardisierte Semantik der EmI (f): } \\
\text { Die EmI sollen im UEDIM in standardisierter Semantik } \\
\text { dargestellt werden, um einen einheitlichen Zugriff auf diese EmI } \\
\text { zu ermöglichen. Im Rahmen der Modellierung des UEDIM sollen } \\
\text { Ontologien einbezogen werden, um EmI in Form von } \\
\text { einheitlichen Terminologien darzustellen [20]. Das UEDIM muss } \\
\text { die eindeutige Definition eines Messpunkttyps (Zuordnung von } \\
\text { Bezeichnung, Datentyp, Maßeinheit, zulässigem Wertebereich } \\
\text { z.B. anhand einer ID wie in [18] dargestellt) enthalten. }\end{array}$ \\
\hline & $\begin{array}{l}1,2,3,4,5,6 \\
7,8,9,10,11 \\
12,13,14,15 \\
16\end{array}$ & $\begin{array}{l}\text { Unterstützung von etablierten tEnMS-Funktionalitäten (f): } \\
\text { Die etablierten tEnMS-Funktionalitäten der bestehenden } \\
\text { Energieprofile [21] [22] [8] sollten unterstützt werden. }\end{array}$ \\
\hline & $1,2,3,4,14$ & $\begin{array}{l}\text { Verwendung der vorhandenen OPC UA Companion } \\
\text { Spezifikationen (f): } \\
\text { Bei der Verwendung des UEDIM muss sichergestellt werden, dass } \\
\text { die etablierten OPC UA Companion Spezifikationen [11] [12] [13] } \\
\text { unterstützt werden, sofern die Quelle der EmI ein Energieprofil } \\
\text { unterstützendes Gerät ist. }\end{array}$ \\
\hline & 1 & $\begin{array}{l}\text { Integration von IIoT-Technologien (nf): } \\
\text { Das UEDIM sollte auf IIoT-Geräten ausführbar sein, damit diese } \\
\text { EmI an die Energiemanagement-Applikation liefern können, wie } \\
\text { in [23] gefordert. }\end{array}$ \\
\hline \multirow[t]{2}{*}{$\begin{array}{l}\text { Schnittstellen zu EmI von der } \\
\text { Feldebene und zur } \\
\text { Energiemanagement- } \\
\text { Applikation }\end{array}$} & $1,2,3,4,14$ & $\begin{array}{l}\text { Austausch von EmI mit der Feldebene (f): } \\
\text { Die EmI von nicht-Ethernet-basierten (z. B. IO-Link) und } \\
\text { Ethernet-basierten Feldgeräten (z. B. Modbus TCP oder } \\
\text { PROFINET) sollten im UEDIM darstellbar sein (siehe Unterkapitel } \\
\text { 2.2). }\end{array}$ \\
\hline & $\begin{array}{l}1,2,4,5,14, \\
15\end{array}$ & $\begin{array}{l}\text { Kompatibilität mit etablierten Kommunikationsschnittstellen } \\
\text { (nf): } \\
\text { Die im UEDIM dargestellten EmI sollten über die etablierten OPC- } \\
\text { UA- oder MQTT-Kommunikationsschnittstellen bereitgestellt }\end{array}$ \\
\hline
\end{tabular}




\begin{tabular}{|c|c|c|}
\hline Anforderungsgruppe & $\begin{array}{l}\text { Zugeordnete } \\
\text { Personas }\end{array}$ & Funktionale (f) und nicht-funktionale (nf) Anforderungen \\
\hline & & $\begin{array}{l}\text { werden, um die jeweiligen Vorteile der Kommunikationssysteme } \\
\text { zu nutzen, wie in Unterkapitel } 2.2 \text { beschrieben. }\end{array}$ \\
\hline \multirow[t]{3}{*}{ Allgemein } & $\begin{array}{l}1,3,4,5,7,13 \\
14,15\end{array}$ & $\begin{array}{l}\text { Reduktion des Engineering-Aufwands (nf): } \\
\text { Die Erstellung von Edge-Device oder SPS-Programmen, die } \\
\text { primär die Bereitstellung der EmI realisieren, ist mit einem } \\
\text { hohen Engineering-Aufwand verbunden [3]. Die Gestaltung des } \\
\text { UEDIM soll darauf abzielen, diesen Engineering-Aufwand zu } \\
\text { reduzieren. Die standardisierte Semantik soll die Anwendung } \\
\text { von Codegeneratoren zur automatischen Generierung von Edge- } \\
\text { Device- oder SPS-Programmen, die zur Bereitstellung von EmI } \\
\text { dienen, begünstigen (vgl. [14]). }\end{array}$ \\
\hline & $2,4,5,14,15$ & $\begin{array}{l}\text { Verarbeitungszeit auf SPSen oder Edge-Devices (nf): } \\
\text { Wenn EmI zwischen der Feldebene und übergeordneten Ebenen } \\
\text { des Automatisierungssystems übertragen werden, muss sich die } \\
\text { Zykluszeit bei Verwendung des UEDIM an branchenüblichen } \\
\text { Zykluszeiten orientieren, die mit vergleichbaren Programmen } \\
\text { (unter Verwendung der OPC UA Companion Spezifikation [11]) } \\
\text { erreicht werden. }\end{array}$ \\
\hline & $\begin{array}{l}1,2,3,4,5,14, \\
15\end{array}$ & $\begin{array}{l}\text { Modularität des UEDIM (nf): } \\
\text { Die Energiemanagementfunktionalitäten sollen einzeln aus dem } \\
\text { tEnMS aktiviert oder deaktiviert werden können [24]. Im UEDIM } \\
\text { müssen daher die Funktionalitätsmodule (Messwerterfassung } \\
\text { oder Stand-by-Management-Funktionen aus den festgelegten } \\
\text { Energieprofilen) getrennt voneinander konzipiert werden, damit } \\
\text { sie unabhängig voneinander sind. }\end{array}$ \\
\hline
\end{tabular}

\section{Fazit und Ausblick}

In diesem Beitrag wurde die Anforderungsermittlung für ein UEDIM vorgestellt, welches die Standardisierung der Semantik von EmI zum Ziel hat. Als Grundlage für die Anforderungsermittlung wurde relevante Literatur zusammengefasst und näher untersucht, von welchen Feldgerätetypen EmI wie z.B. Energiezählwerte bereitgestellt werden können. Hinzukommend wurde vorgestellt, über welche Kommunikationstechnologien die EmI bis zur Energiemanagement-Applikation weitergeleitet werden können und welche Vor- und Nachteile die jeweiligen Kommunikationstechnologien aufweisen. Um Ansätze für eine standardisierte Semantik von EmI aufzugreifen, werden außerdem aktuelle Ansätze wie Energieprofil-spezifische OPC UA-Companion Spezifikationen betrachtet. Im Rahmen der Anforderungsermittlung wurde mit einer Persona-Analyse beschrieben, welche Stakeholder von der Einführung eines UEDIM profitieren würden und welche Interessengruppen identifiziert werden können. Auf der Basis der Literaturrecherche und der Persona-Analyse wurden Anforderungen an ein UEDIM aufgestellt.

In den nächsten Schritten des Forschungsprojekts werden die Energieprofil-spezifischen Datenmodelle gegenübergestellt und die Funktionsumfänge im Detail verglichen. Auf diesem Weg werden die Energiemanagement-Funktionalitäten etablierter Kommunikationstechnologien erfasst und können in die Konzeption des UEDIM eingebracht werden. Auf dieser Grundlage und auf Basis der durchgeführten Anforderungsermittlung wird in einem iterativen Vorgehen ein UEDIM modelliert. 


\section{Danksagung}

Die Autoren bedanken sich für die finanzielle Unterstützung im Rahmen des IGF-Projekts 21329 N, das vom Bundesministerium für Bundesministerium für Wirtschaft und Energie (BMWi) auf der Grundlage eines Beschlusses des Deutschen Bundestages gefördert wird.

\section{Literatur}

[1] 2018. ISO 50001:2018-08 - Energy management systems - Requirements with guidance for use

[2] Würger, A., Niemann, K.-H. u. Fay, A.: Concept for an Energy Data Aggregation Layer for Production Sites. A combination of AutomationML and OPC UA. IEEE 23rd International Conference on Emerging Technologies And Factory Automation (ETFA). 2018, S. 1051-1055

[3] Würger, A.: Automatisierte Generierung von Energiemanagementfunktionen auf der Basis des PROFINETEnergieprofils. Dissertation, Helmut-Schmidt-Universität / Universität der Bundeswehr Hamburg 2020

[4] L.-T. Reiche, M. Runge, A. Fay u. K.-H. Niemann: Communication of energy data in automation systems. 17th IEEE International Conference on Factory Communication Systems (WFCS). 2021

[5] M. Runge, L.-T. Reiche, K.-H. Niemann, A. Fay u. A. Würger: Requirements for an energy data information model for a communication-independent device description. 26th IEEE International Conference on Emerging Technologies and Factory Automation (ETFA). 2021

[6] Common Application Profile PROFIenergy, PROFIBUS Nutzerorganisation e. V., 2021

[7] SERCOS International e.V.: sercos Energy - Spezifikation des Energieprofils für sercos III verfügbar. https://www.sercos.de/news-events/newsdetail/sercos-energy-spezifikation-des-energieprofils-fuer-sercosiii-verfuegbar/, abgerufen am: 25.11.2021

[8] ODVA: CIP Energy. https://www.odva.org/technology-standards/distinct-cip-services/cip-energy/, abgerufen am: 01.06.2021

[9] OPC Foundation: Namespace and identifiers for OPC UA for PROFIenergy Information Model. https://reference.opcfoundation.org/v104/PNEM/v100/docs/A.1/, abgerufen am: 06.12.2021

[10] IEC 62541-3:2015. IEC 62541-3:2020 - OPC Unified Architecture - Part 3: Address Space Model

[11] Profibus Nutzerorganisation e. V.: OPC UA for Energy Management, 2020. https://de.profibus.com/downloads/opc-ua-for-energy-management-companion-specification, abgerufen am: 01.06 .2021

[12] OPC Foundation: OPC Unified Architecture for Sercos Devices, 2017. https://opcfoundation.org/developertools/specifications-opc-ua-information-models/opc-unified-architecture-for-sercos-devices/, abgerufen am: 02.12.2021

[13] ODVA: OPC UA Companion Specification to Be Developed for CIP. https://www.odva.org/news/opc-ua-companion-specification-to-be-developed-for-cip/, abgerufen am: 02.12.2021

[14] Würger, A., Niemann, K.-H., Fay, A., Gienke, M. u. Paulick, M.: Integriertes Anlagenengineering zur Erhöhung der Energieeffizienz. atp Edition

[15] A. Veichtlbauer, M. Ortmayer u. T. Heistracher: OPC UA integration for field devices. In: IEEE 15th International Conference on Industrial Informatics (INDIN)

[16] MQTT, OPC UA und PROFINET für IIoT, Leßmann, G. u. Schriegel, S., 2021

[17] Profanter, S., Tekat, A., Dorofeev, K., Rickert, M. u. Knoll, A.: OPC UA versus ROS, DDS, and MQTT: Performance Evaluation of Industry 4.0 Protocols. 20th 2019 IEEE International Conference on Industrial Technology (ICIT). IEEE 2019 - 2019, S. 955-962

[18] NAMUR Open Architecture - NOA Informationsmodell, NE 176, Interessengemeinschaft Automatisierungstechnik der Prozessindustrie, 2021

[19] Nielsen, L.: Personas - User Focused Design. 2013

[20] Linnenberg, T., Mueller, A. W., Christiansen, L. J., Seitz, C. W. u. Fay, A.: Ontoenergy - A lightweight ontology for supporting energy-efficiency tasks: Enabling generic evaluation of energy efficiency in the engineering phase of automated manufacturing plants. 5th International Conference on Knowledge Engineering and Ontology Development (KEOD). 2013, S. 337-344

[21] Common Application Profile PROFIenergy, PROFIBUS Nutzerorganisation e. V., 2019

[22] SERCOS International e.V.: Energy Profile, 2011. https://www.sercos.de/news-events/newsdetail/sercos-energy-spezifikation-des-energieprofils-fuer-sercos-iii-verfuegbar/, abgerufen am: 16.06.2021 
[23] Al-Gumaei, K., Schuba, K., Friesen, A., Heymann, S., Pieper, C., Pethig, F. u. Schriegel, S.: A Survey of Internet of Things and Big Data Integrated Solutions for Industrie 4.0. IEEE 23rd International Conference on Emerging Technologies And Factory Automation (ETFA). 2018, S. 1417-1424

[24] Javied, T., Huprich, S. u. Franke, J.: Cloud based Energy Management System Compatible with the Industry 4.0 Requirements. IFAC-PapersOnLine 52 (2019) 10, S. 171-175 